\title{
Small nucleolar host gene 6 promotes esophageal squamous cell carcinoma cell proliferation and inhibits cell apoptosis
}

\author{
RUI-HUA FAN $^{1 *}$, JIA-NI GUO $^{1 *}$, WEI YAN ${ }^{2 *}$, MING-DE HUANG $^{1}$, \\ CHANG-LI ZHU ${ }^{1}$, YONG-MEI YIN ${ }^{3}$ and XIAO-FEI CHEN ${ }^{1}$ \\ Departments of ${ }^{1}$ Medical Oncology and ${ }^{2}$ Gastroenterology, Huai'an First People's Hospital, \\ Nanjing Medical University, Huai'an, Jiangsu 223300; ${ }^{3}$ Department of Oncology, The First Affiliated Hospital of \\ Nanjing Medical University, Jiangsu Province Hospital, Nanjing, Jiangsu 210029, P.R. China
}

Received June 22, 2016; Accepted November 21, 2017

DOI: $10.3892 / \mathrm{ol} .2018 .8087$

\begin{abstract}
Esophageal cancer (ESCC) is one of the most common causes of cancer-associated mortality in China. The present investigation reveals that non-coding RNAs (ncRNAs), including long ncRNAs (lncRNAs), exert a significant effect on the initiation, development and metastasis of malignant tumors, including ESCC. However, to the best of our knowledge, the function of non-protein-coding genes that host small nucleolar RNAs has not been investigated in cancer, particularly in ESCC. The expression of small nucleolar host gene 6 (SNHG6) in 70 ESCC tissues and paired adjacent tissues was measured by reverse transcription quantitative polymerase chain reaction. Analysis demonstrated that SNHG6 expression was significantly increased in ESCC tissues, and associated with tumor size $(\mathrm{P}=0.040)$ and Tumor-Node-Metastasis stage $(\mathrm{P}<0.01)$. Knockdown of SNHG6 may inhibit proliferative and colony-forming abilities, and induce apoptosis, in ESCC cells. To the best of our knowledge, the data from the present study indicated for the first time that SNHG6 was upregulated in ESCC tissues and cell lines. This novel lncRNA may exert
\end{abstract}

Correspondence to: Dr Yong-Mei Yin, Department of Oncology, The First Affiliated Hospital of Nanjing Medical University, Jiangsu Province Hospital, 300 Guangzhou Road, Nanjing, Jiangsu 210029, P.R. China

E-mail: ym.yin@hotmail.com

Dr Xiao-Fei Chen, Department of Medical Oncology, Huai'an First People's Hospital, Nanjing Medical University, 1 West Huanghe Road, Huai'an, Jiangsu 223300, P.R. China

E-mail: cxf003835@126.com

*Contributed equally

Abbreviations: lncRNA, long non-coding RNA; ESCC, esophageal squamous cell carcinoma; RT-qPCR, reverse transcription-quantitative polymerase chain reaction

Key words: esophageal squamous cell carcinoma, non-coding RNA, small nucleolar host gene 6 , malignant proliferation a marked effect on the generation and progression of ESCC, potentially providing a novel perspective on ESCC diagnosis and management.

\section{Introduction}

Esophageal cancer (ESCC) has become one of the leading causes of cancer-associated mortality in China over the past few decades (1). Despite the marked improvements made in chemotherapy and radiotherapy in the past few decades, metastases and tumor recurrence in ESCC remain associated with a poor prognosis (2). To improve patient survival, there is a pressing requirement to understand the mechanism of pathogenesis and to identify novel biomarkers and therapeutic targets in patients with ESCC. One potential approach to cancer diagnosis and treatment is through the assessment and targeting of non-coding RNAs (ncRNAs), which are widely transcribed in the eukaryotic genome (2). Long ncRNAs (lncRNAs), which have become a focus of the study of ncRNAs, are a class of non-coding RNAs characterized by a length of $>200$ nucleotides (2). Evidence indicates that lncRNAs may exhibit a marked effect on a number of molecular genetics and cellular processes, including chromatin modification, cellular differentiation and cell cycle regulation (3). Concurrently, lncRNAs have been demonstrated to be dysregulated in various tumors and to serve as promoters or suppressors in multiple signaling pathways (4). Previous studies have indicated that lncRNAs are crucial regulators of pathways involved in tumorigenesis and the progression of ESCC, including metastasis-associated lung adenocarcinoma transcript 1 (MALAT1) (5), sprouty RTK signaling antagonist 4-intronic transcript 1 (SPRY4-IT1) (6) and colon cancer-associated transcript 2 (7). A previous study demonstrated that lncRNA MALAT1 expression was increased in ESCC tissues compared with adjacent normal tissues (5). MALAT1 knockdown may inhibit ESCC cell proliferation, migration and tumor-sphere formation, but increase cell apoptosis (5). Downregulation of MALAT1 decreased the expression of $\beta$-catenin, Lin 28 homolog A and enhancer of zeste 2 polycomb repressive complex 2 subunit (Ezh2), whereas overexpression of Ezh2 reversed the small interfering (si)-MALAT1-mediated repression (5). In addition, Zhang et al (8) identified that the expression of lncRNA 
SPRY4-IT1 was increased in ESCC cell lines compared with normal esophageal epithelial cells. Overexpression of SPRY4-IT1 may increase ESCC cell motility via induction of the epithelial-mesenchymal transition (8). On the contrary, other studies also revealed that several lncRNAs may serve as tumor suppressor genes in ESCC, including the lncRNAs low expression in tumor (9) and urothelial cancer associated 1 (10). However, despite the progress made in understanding lncRNAs, the non-protein-coding genes encoding small nucleolar RNAs (snoRNAs) have received little attention.

snoRNAs, which are ncRNAs, are small RNAs measuring 60-300 nucleotides in length (11). Owing to their nucleolar localization, the majority of snoRNAs serve as guide RNAs for post-transcriptional modifications, to ensure the production of efficient and accurate ribosomes (12). It was initially assumed that the non-coding genes encoding snoRNAs have no function, but may host coding sequences in their introns (13). However, previous studies have indicated that snoRNA host genes (SNHGs) may serve critical roles in cancer, with roles documented for SNHG1 in non-small cell lung cancer (14), SNHG3 in hepatocellular carcinoma (15) and SNHG12 in human osteosarcoma (16). A previous study by Makarova and Kramerov (17) proposed the existence of the unusual snoRNA gene small nucleolar host gene 6 (U87HG), also termed SNHG6, which is a housekeeping gene of the 5'-terminal oligopyrimidine tract (TOP) family and associated with ribosomes. SNHG6 demonstrated a high degree of conservation and was ineffectively degraded by nonsense-mediated mRNA decay (NMD), which indicated that it served additional functions separate from the production of U87 RNA (17). The present study used reverse transcription-quantitative polymerase chain reaction (RT-qPCR), MTT assays, flow cytometry and subcellular fraction assays to investigate the expression and functional roles of lncRNA SNHG6 in ESCC. The results indicated that the expression of SNHG6 was significantly upregulated in ESCC tissues and cell lines compared with normal esophageal epithelial cells. Inhibition of SNHG6 may result in diminished cell growth and increased apoptosis, which indicates the potential role served by SNHG6 in ESCC.

\section{Materials and methods}

Tissue samples and patient data. Patients $(\mathrm{n}=70$; age range, 24-84; median age, 64; admitted between January and December 2012) diagnosed with primary ESCC and scheduled for routine surgery at Huai'an First People's Hospital, Nanjing Medical University (Huai'an, China) were included in the present study. All the patients with a histological diagnosis had not received preoperative therapy. Clinical information, including age, gender, history of smoking and drinking, tumor size and pathological Tumor-Node-Metastasis (TNM) stage [according to the 7th AJCC TNM staging system (18)], was collected from clinical data and personal interviews. ESCC and adjacent non-cancerous tissues following resection were snap-frozen in liquid nitrogen and stored at $-80^{\circ} \mathrm{C}$. All procedures performed in the present study involving human participants were conducted in accordance with the ethical standards of Huai'an First People's Hospital, Nanjing Medical University and with the 1964 Helsinki declaration and its later amendments. Informed consent was obtained from all individual participants included in the present study.

Cell culture. The ESCC ECA-109 and TE-1 cell lines were purchased from the Type Culture Collection of the Chinese Academy of Sciences (Shanghai, China) and cultured in high-glucose Dulbecco's modified Eagle's medium (Gibco; Thermo Fisher Scientific, Inc., Waltham, MA, USA) containing, penicillin-streptomycin and $10 \%$ fetal bovine serum (FBS; Gibco; Thermo Fisher Scientific, Inc.). A normal human esophageal epithelial cell line (HEEC) was obtained from ScienCell Research Laboratories, Inc. (San Diego, CA, USA) and grown in RPMI-1640 medium (Gibco; Thermo Fisher Scientific, Inc.), containing penicillin-streptomycin and $10 \%$ FBS. All cells were maintained in humidified incubators under standard conditions $\left(37^{\circ} \mathrm{C}, 5 \% \mathrm{CO}_{2}\right)$.

Cell transfection. ECA-109 and TE-1 cells were transfected with specific small interfering RNA (siRNA) oligonucleotides. A total of 3 different siRNAs (sequences are listed in Table I) were designed to ensure the efficiency of interference, 2 of which (si-SNHG6-2\# and si-SNHG6-3\#) were considered appropriate for SNHG6 knockdown as they had an interference efficiency of $>70 \%$ (Invitrogen; Thermo Fisher Scientific, Inc.). Negative control siRNA (si-NC) was also purchased from Invitrogen; Thermo Fisher Scientific, Inc. ESCC cells were seeded at 6-well plates for $24 \mathrm{~h}$. Cells were then transfected with either SNHG6-siRNA (100 nM) or si-NC (100 nM) using Lipofectamine RNAiMAX transfection reagent ${ }^{\circledR}$ (Invitrogen; Thermo Fisher Scientific, Inc.), according to the manufacturer's protocol. Following incubation for $48 \mathrm{~h}$, transfected cells were used for further experiments.

RNA extraction and reverse transcription quantitative polymerase chain reaction $(R T-q P C R)$ analysis. Total RNA was extracted from tissues specimens or cultured cells with TRIzol ${ }^{\circledR}$ reagent (Life Technologies; Thermo Fisher Scientific, Inc.) according to the manufacturer's protocol. A total of $1 \mu \mathrm{g}$ total RNA was used for the reverse transcription reaction in a final volume of $20 \mu \mathrm{l}$, using random primers from the PrimeScript RT Reagent kit with gDNA Eraser (Takara Biotechnology Co., Ltd., Dalian, China) and $1 \mu \mathrm{l}$ cDNA was used, according to the manufacturer's protocols, for subsequent RT-qPCR reactions (SYBR Premix Ex Taq, Takara Bio, Inc., Otsu, Japan) of denaturation at $95^{\circ} \mathrm{C}$ for $5 \mathrm{sec}$, annealing at $60^{\circ} \mathrm{C}$ for $34 \mathrm{sec}$, elongation at $68^{\circ} \mathrm{C}$ for $20 \mathrm{sec}$ for 40 cycles. The constitutively expression gene GAPDH was used to normalize target gene expression and U1 was used to indicate nuclear expression. The RT-qPCR analysis was performed on ABI 7500 Real-Time PCR system using the $2^{-\Delta \Delta C t}$ method (19) (Applied Biosystems; Thermo Fisher Scientific, Inc.). The assay was run in triplicate for each sample. The primer sequences are summarized in Table I. The specimens were divided into a high-expression group $(\mathrm{n}=50)$ and a low-expression group $(n=20)$ according to the expression of SNHG6 (a fold-change $\geq 1$ represents high expression of SNHG6, while a fold-change $<1$ indicates low expression of SNHG6 in ESCC tissues.).

Cell proliferation assays. Each cell line was seeded in flat-bottomed 96-well plates (3,000 cells/well) $24 \mathrm{~h}$ following 
siRNA transfection. The absorbance was measured using a microplate reader at a wavelength of $490 \mathrm{~nm}$. Cell proliferation was evaluated using an MTT Cell Proliferation Reagent kit I (Roche Diagnostics, Basel, Switzerland). For the colony-formation assay, 500 transfected cells were plated in a 6-well plate and maintained in RPMI-1640 containing 10\% FBS. After 14 days, cells were washed twice with PBS, then fixed with $4 \%$ methanol at room temperature for $15 \mathrm{~min}$ and stained with $0.1 \%$ crystal violet (dissolved using $95 \%$ ethanol) at room temperature for $15 \mathrm{~min}$ (Sigma-Aldrich; Merck KGaA, Darmstadt, Germany). The colony formation was determined by counting the number of stained colonies under a light Olympus microscope (x40). All the experiments were performed in triplicate. The formula for the colony formation rate was as follows: Rate $(\%)=$ numbers of colony/initial cell population x 100 .

Cell apoptosis analysis. Subsequent to transfection with si-SNHG6 or si-NC, cells were harvested using centrifugation $\left(4^{\circ} \mathrm{C}\right.$ at $7,500 \times \mathrm{g}$ for $\left.5 \mathrm{~min}\right)$ and stained using an annexin V-Fluorescein Isothiocyanate/Propidium Iodide Apoptosis Detection kit (BD Biosciences, Franklin Lakes, NJ, USA). A flow cytometry system (FACScan ${ }^{\circledR}$, BD Biosciences) equipped with CellQuest Pro Software version 5.1 (BD Biosciences, USA) was applied to analyze cell apoptosis, in accordance with the manufacturer's protocol. Cells were divided into viable cells, dead cells, early apoptotic cells and late apoptotic cells. The relative proportion of early apoptotic cells and apoptotic cells were expressed as the mean \pm standard deviation.

Subcellular fractionation. The separation of the nuclear and cytosolic fractions of ECA-109 and TE-1 cells was performed using a PARIS ${ }^{\mathrm{TM}}$ kit (Life Technologies; Thermo Fisher Scientific, Inc.), according to the manufacturer's protocol.

Statistical analysis. SPSS 19.0 software (IBM Corp., Armonk, NY, USA) was used to perform statistical analyses. All experiments were performed in triplicate. Student's t-test was performed to evaluate the difference in SNHG6 expression between ESCC and adjacent non-cancerous tissues and was used to compare two independent groups. One-way analysis of variance, followed by a Tukey's post hoc test, was used to compare three or more groups. The association between SNHG6 expression and clinicopathological features was evaluated using Pearson's $\chi^{2}$ test. $\mathrm{P}<0.05$ was considered to be indicate a statistically significant difference.

\section{Results}

SNHG6 is upregulated in ESCC tissues and associated with clinicopathological features. SNHG6 expression was analyzed in 70 human ESCC specimens and matched non-cancerous tissues by RT-qPCR. The expression level of SNHG6 was significantly increased in ESCC tissues compared with matched adjacent tissues (50 of the 70 tissues, an increase of $\geq 1.0$-fold; $\mathrm{P}<0.01$; Fig. 1A and B). Subsequently, the specimens were divided into a high-expression group $(n=50)$ and a low-expression group $(n=20)$ according to the expression of SNHG6 (a fold-change $\geq 1$ represents high expression of SNHG6, while a fold-change $<1$ indicates low expression of
Table I. Sequence for primers and siRNAs.

Experimental

method

Sequence, 5'-3'

\begin{tabular}{ll}
\hline $\begin{array}{l}\text { RT-qPCR } \\
\text { GAPDH }\end{array}$ & \\
Forward & GGGAGCCAAAAGGGTCAT \\
Reverse & GAGTCCTTCCACGATACCAA \\
SNHG6 & \\
Forward & TTAGTCATGCCGGTGTGGTG \\
Reverse & AATACATGCCGCGTGATCCT \\
U1 & \\
Forward & GGGAGATACCATGATCACGAAGGT \\
Reverse & CCACAAATTATGCAGTCGAGTTTCCC \\
Transfection & \\
siRNA & \\
oligonucleotides & \\
si-NC & UUCUCCGAACGUGUCACGUTT \\
si- SNHG6-1\# & UUCACCUCAAAGGCUUUCUUGCACC \\
si- SNHG6-2\# & AAAUGCUGCAUGCCACACUUGAGGU \\
si- SNHG6-3\# & GCGGCAUGUAUUGAGCAUAUAGGUU
\end{tabular}

RT-qPCR, reverse transcriptase-quantitative polymerase chain reaction; F, forward; R, reverse; siRNA, small interfering RNA; SNHG6, small nucleolar host gene 6; AFAP1-AS1, actin filament associated protein 1 antisense RNA 1.

SNHG6 in ESCC tissues.). Furthermore, the prognostic role of SNHG6 in determining the clinical significance of patients with ESCC was investigated by analyzing the association between SNHG6 expression and clinicopathological features (Table II). The data indicated that SNHG6 expression was associated with tumor size $(\mathrm{P}=0.040)$ and TNM stage $(\mathrm{P}<0.01)$, but was not associated with other parameters.

Effect of SNHG6 on cell proliferation. Since SNHG6 was also upregulated in ESCC ECA109/TE-1 cell lines compared with expression in normal esophageal epithelial HEEC cells (Fig. 1C), further investigation was undertaken into the functional role of SNHG6 in ESCC. To evaluate the role of SNHG6 in maintaining the malignant phenotypes of ESCC cells, ECA109/TE-1 cells were transfected with si-SNHG6-2\# or si-SNHG6-3\#, prior to being used for subsequent experiments. The MTT assay demonstrated that the proliferation of ECA109/TE-1 cells was markedly inhibited by the knockdown of SNHG6 (Fig. 2A and B). In addition, colony formation assays were performed to assess the effect of SNHG6 on ESCC cells, and indicated that clonogenic survival was markedly decreased following SNHG6 knockdown in ECA109 and TE1 cell lines (Fig. 2C and D).

Effect of SNHG6 on cell apoptosis. To examine the effect of SNHG6 on cell apoptosis, flow cytometry analysis was utilized. The results of this analysis indicated that the inhibition of SNHG6 markedly induced cell apoptosis compared with the si-NC group (Fig. 2E and F). 
A

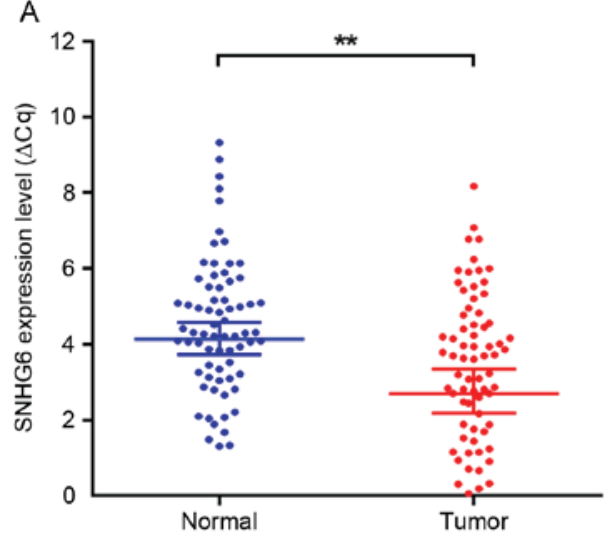

C

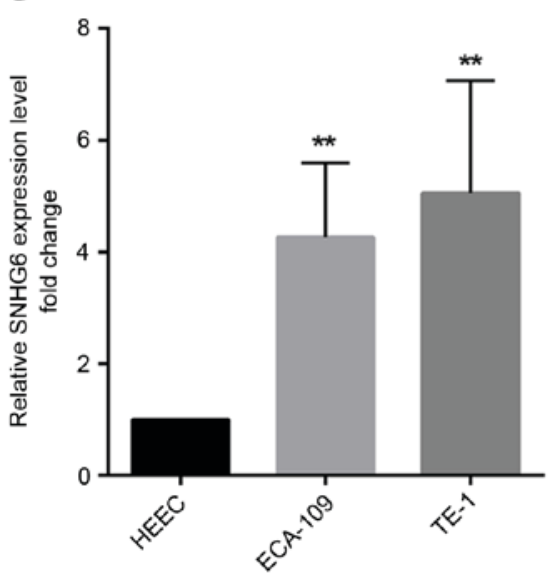

B

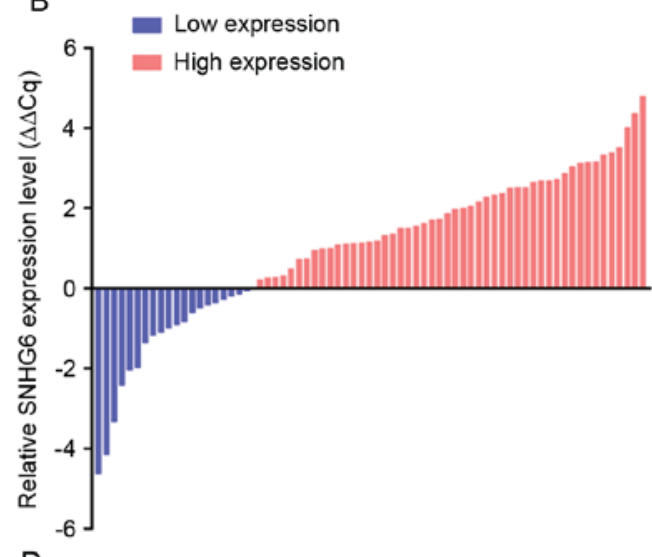

D

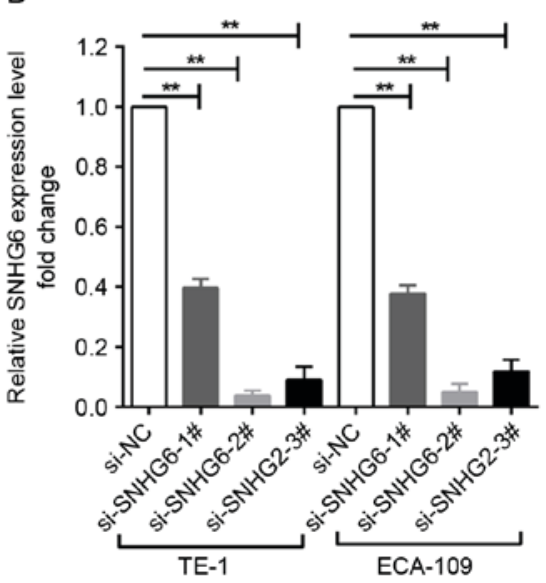

Figure 1. Relative expression of SNHG6 in ESCC tissues. (A) RT-qPCR was used to detect the relative expression of SNHG6 in ESCC tissues and adjacent normal tissues $(\mathrm{n}=70)$. The results were normalized to GAPDH expression and presented as $\triangle \mathrm{Cq}$. (B) Relative SNHG6 expression in ESCC tissues and adjacent normal tissues $(n=70)$. A fold-change $\geq 1$ represents high expression of SNHG6, while a fold-change $<1$ indicates low expression of SNHG6 in ESCC tissues. (C) Relative SNHG6 expression in 2 ESCC cell lines (ECA-109 and TE-1) and 1 normal esophageal epithelium cell line (HEEC). (D) RT-qPCR was used to assess the expression of SNHG6 in ECA-109 and TE-1 cell lines transfected with different si-SNHG6 sequences. ${ }^{* *}$ P $<0.01$ with comparisons shown by lines. RT-qPCR, reverse transcriptase-quantitative polymerase chain reaction; ESCC, esophageal squamous cell carcinoma; si-SNHG6, small interfering RNA targeting small nucleolar host gene 6; Cq, quantification cycle; NC, negative control.

Subcellular localization of SNHG6. Following separation of ECA-109 and TE-1 cells, RNA was isolated from the nuclear and cytosolic fractions of the cells and SNHG6 expression was measured by RT-qPCR. GAPDH was used as a reference cytoplasm indicator and U1 was used as a reference nucleus indicator. The results revealed that, in the 2 cell lines, the expression level of SNHG6 was significantly increased in the cytoplasm compared with the nucleus (Fig. 2G and H).

\section{Discussion}

ncRNAs, a class of genetic, epigenetic and translational regulators without protein-coding capacity, contain short and long transcripts and have the potential to be used as biomarkers for multiple diseases (20). IncRNAs, which are $>200$ nucleotides, are members of the ncRNA family. It has been demonstrated in a variety of types of cancer that the expression of lncRNAs is closely associated with the carcinogenesis, disease development, metastasis and prognosis of patients with malignancy (4). IncRNAs may become targets for cancer diagnosis and treatment.

A subset of small ncRNAs, snoRNAs, in the nucleoli are involved in multiple steps of rRNA processing (21). According to their structure, snoRNAs may be divided into 2 classes: C/D box snoRNAs, which serve as guide RNAs in site-specific 2'-O-methylation of rRNAs, and H/ACA box snoRNAs, which direct site-specific pseudouridylation of rRNAs (20). In vertebrates, the majority of snoRNA genes reside within the introns of protein-coding genes and encode nucleolar proteins or proteins involved in translation (22). However, a small number of SNHGs contain multiple stop codons and do not code for proteins, including UHG (23), U17HG (24), U19HG (25), gas5 (13) and U50HG (26). In 2005, a study reported the existence of an unusual snoRNA host gene SNHG6, also termed U87HG, a novel lncRNA 472 nucleotides in length and located within an intron of a novel non-protein-coding gene (17). SNHG6 is a housekeeping gene of the 5'TOP family and is associated with ribosomes. The degree of conservation of SNHG6 RNA is similar to those of the untranslated regions of protein-coding genes. Additionally, SNHG6 RNA is ineffectively degraded by NMD. NMD detects the mRNAs that contain premature termination codons and triggers their degradation to prevent the accumulation of truncated and potentially harmful proteins. Concomitant with the production of U87 RNA, SNHG6 may also participate in translation or its regulation (17). SNHG6 may be functionally important, owing 
Table II. Association between SNHG6 expression and clinicopathological characteristics in esophageal squamous cell carcinoma.

SNHG6 expression

Clinical parameters

High (fold-change $\geq 1$ )

Low (fold-change <1)

P-value

Age, years

$\leq 64$

25

0.449

$>64$

25

12

Sex

Male

Female

Drinking state

Yes (frequent drinkers)

No (never drinkers)

Tumor size, $\mathrm{cm}$

14

$\leq 3$

$>5$

Tumor stage

2

II

27

III

21

10

7

1

$<0.01$

${ }^{a} \chi^{2}$ P-value. SNHG6, small nucleolar host gene 6 .

A

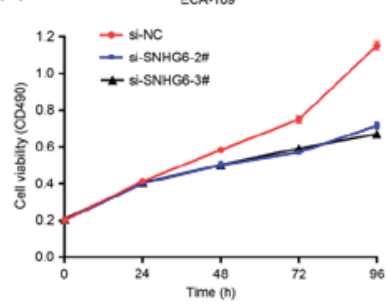

E

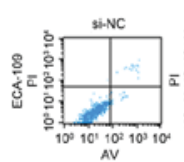

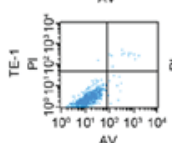

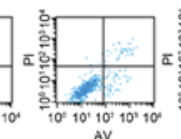

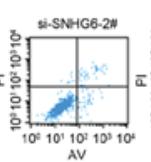
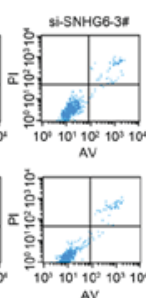

B

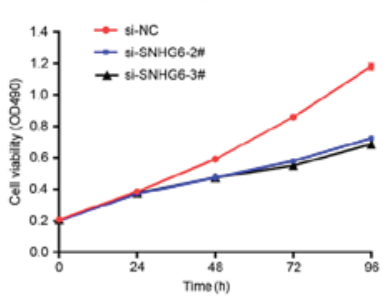

F

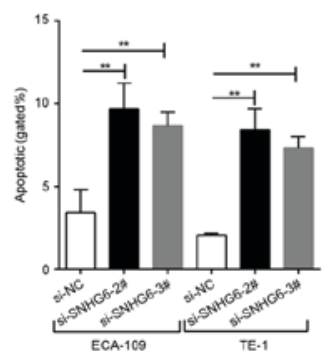

C

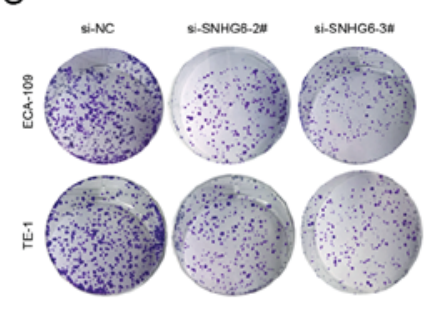

G

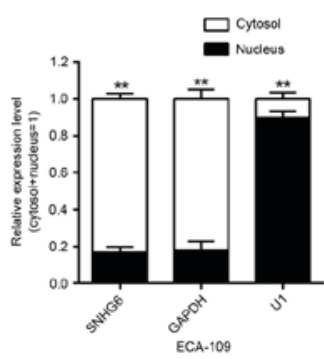

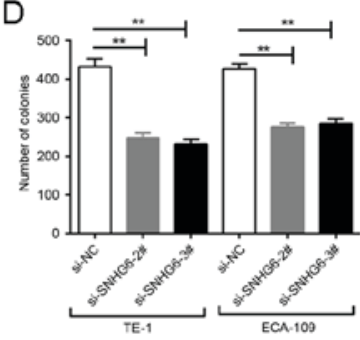

$\mathrm{H}$

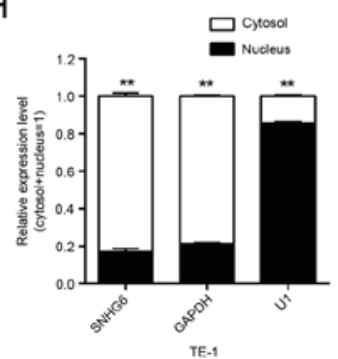

Figure 2. Knockdown of SNHG6 inhibited proliferation and induced apoptosis in ESCC cells, and triggered the subcellular localization of SNHG6 in ESCC cells. Effect of SNHG6 knockdown on cell viability in vitro was measured by MTT assays in (A) ECA-109 and (B) TE-1 cells. Effect of SNHG6 knockdown on cell proliferation in vitro was measured by (C) colony-forming assays, followed by (D) quantification of these results. Effect of SNHG6 knockdown on cell apoptosis in vitro was measured by (E) flow cytometry assays, followed by (F) quantification of these results. Subcellular fraction assay revealed that SNHG6 was located in the cytosol rather than the nucleus in $(\mathrm{G}) \mathrm{ECA}-109$ and $(\mathrm{H}) \mathrm{TE}-1$ cells. ${ }^{* *} \mathrm{P}<0.01$ comparisons between the cytosol and the nucleus. si-SNHG6, small interfering RNA targeting small nucleolar host gene 6; NC, negative control; PI, propidium iodide; AV, annexin V.

to the areas of high local similarity to U87 RNA interspersed among the loosely-conserved sequence it contains (17).
In the present study, the function of SNHG6 in ESCC, and the potential association between its expression and 
clinicopathological features was examined. Data analysis indicated that the upregulation of SNHG6 was associated with tumor size and TNM stage. The expression level of SNHG6 in ECA109 and TE-1 cells was significantly increased compared with that in HEEC cells. Induced downregulation of SNHG6 markedly inhibited the proliferative and colony-forming abilities of, and induced apoptosis in, ESCC cells, indicating its oncogenic role. This result indicated the critical role of SNHG6 in tumorigenesis and the progression of ESCC. Additional experimental results demonstrated that SNHG6 RNA was predominately localized in the cytoplasm, which was consistent with data from Makarova and Kramerov (17). As it appears that the regulatory mechanisms of lncRNAs are usually associated with their localization (4), it can be assumed that SNHG6 may serve as a microRNA sponge, which are able to bind with several miRNAs to inhibit their expression and their downstream pathways, or as an RNA-binding protein to regulate the expression of target genes and participate in multiple pathways, contributing to its oncogenic role in ESCC. Chaudhry (27) suggested that SNHG6 may be involved in the ionizing radiation (IR)-induced stress response in a tumor protein p53 (p53)-dependent manner. Similarly, a previous study indicated that sno-microRNA-28, which directly targets the p53-stabilizing gene TATA-box binding protein associated factor $9 \mathrm{~b}$, was transcriptionally repressed by p53 through SNHG1 (28). These molecules form a regulatory loop that affects p53 stability and downstream p53-regulated pathways (28). Furthermore, Zhao et al (29) identified that SNHG5 may affect acetylation by trapping metastasis-associated 1 family member 2 in the cytosol. SNHG5 overexpression may significantly increase the acetylation levels of histone $\mathrm{H} 3$ and $\mathrm{p} 53$, thereby interfering with the formation of the nucleosome remodeling and histone deacetylation complex (29). Therefore, it is possible that SNHG6 may also affect the cell cycle and apoptosis through p53-associated pathways.

In conclusion, the present study revealed that SNHG6 may exhibit an important effect on the oncogenesis and development of ESCC. As a potential predictor and a promising alternative therapeutic target for future ESCC treatment, the detailed molecular mechanism of SNHG6 involved in ESCC should be clarified by additional studies.

\section{References}

1. Siegel RL, Miller KD and Jemal A: Cancer statistics, 2015. CA Cancer J Clin 65: 5-29, 2015.

2. Deng G and Sui G: Noncoding RNA in oncogenesis: A new era of identifying key players. Int J Mol Sci 14: 18319-18349, 2013.

3. Clark MB and Mattick JS: Long noncoding RNAs in cell biology. Semin Cell Dev Biol 22: 366-376, 2011.

4. Prensner JR and Chinnaiyan AM: The emergence of lncRNAs in cancer biology. Cancer Discov 1: 391-407, 2011.

5. Wang W, Zhu Y, Li S, Chen X, Jiang G, Shen Z, Qiao Y, Wang L, Zheng $\mathrm{P}$ and Zhang Y: Long noncoding RNA MALAT1 promotes malignant development of esophageal squamous cell carcinoma by targeting $\beta$-catenin via Ezh2. Oncotarget 7: 25668-25682, 2016.

6. Cui F, Wu D, He X, Wang W, Xi J and Wang M: Long noncoding RNA SPRY4-IT1 promotes esophageal squamous cell carcinoma cell proliferation, invasion, and epithelial-mesenchymal transition. Tumour Biol 37: 10871-10876, 2016.
7. Zhang X, Xu Y, He C, Guo X, Zhang J, He C, Zhang L, Kong M, Chen B and Zhu C: Elevated expression of CCAT2 is associated with poor prognosis in esophageal squamous cell carcinoma. J Surg Oncol 111: 834-839, 2015.

8. Zhang CY, Li RK, Qi Y, Li XN, Yang Y, Liu DL, Zhao J, Zhu DY, Wu K, Zhou XD and Zhao S: Upregulation of long noncoding RNA SPRY4-IT1 promotes metastasis of esophageal squamous cell carcinoma via induction of epithelial-mesenchymal transition. Cell Biol Toxicol 32: 391-401, 2016.

9. Wang PL, Liu B, Xia Y, Pan CF, Ma T and Chen YJ: Long non-coding RNA-Low Expression in Tumor inhibits the invasion and metastasis of esophageal squamous cell carcinoma by regulating p53 expression. Mol Med Rep 13: 3074-3082, 2016.

10. Wang X, Gao Z, Liao J, Shang M, Li X, Yin L, Pu Y and Liu R: lncRNA UCA1 inhibits esophageal squamous-cell carcinoma growth by regulating the Wnt signaling pathway. J Toxicol Environ Health A 79: 407-418, 2016.

11. Williams GT and Farzaneh F: Are snoRNAs and snoRNA host genes new players in cancer? Nat Rev Cancer 12: 84-88, 2012.

12. Decatur WA and Fournier MJ: rRNA modifications and ribosome function. Trends Biochem Sci 27: 344-351, 2002.

13. Smith CM and Steitz JA: Classification of gas5 as a multi-small-nucleolar-RNA (snoRNA) host gene and a member of the 5'-terminal oligopyrimidine gene family reveals common features of snoRNA host genes. Mol Cell Biol 18: 6897-6909, 1998.

14. You J, Fang N, Gu J, Zhang Y, Li X, Zu L and Zhou Q: Noncoding RNA small nucleolar RNA host gene 1 promote cell proliferation in nonsmall cell lung cancer. Indian J Cancer 3 (Suppl 51): e99-e102, 2014

15. Zhang T, Cao C, Wu D and Liu L: SNHG3 correlates with malignant status and poor prognosis in hepatocellular carcinoma. Tumour Biol 37: 2379-2385, 2016.

16. Ruan W, Wang P, Feng S, Xue Y and Li Y: Long non-coding RNA small nucleolar RNA host gene 12 (SNHG12) promotes cell proliferation and migration by upregulating angiomotin gene expression in human osteosarcoma cells. Tumour Biol 37: 4065-4073, 2016.

17. Makarova JA and Kramerov DA: Noncoding RNA of U87 host gene is associated with ribosomes and is relatively resistant to nonsense-mediated decay. Gene 363: 51-60, 2005.

18. Edge SB, Byrd DR and Compton CC: eds. AJCC Cancer Staging Manual. 7th edition. New York, Springer, 2009.

19. Livak KJ and Schmittgen TD: Analysis of relative gene expression data using real-time quantitative PCR and the 2(-Delta Delta C(T)) method. Methods 25: 402-408, 2001.

20. Busch A, Eken SM and Maegdefessel L: Prospective and therapeutic screening value of non-coding RNA as biomarkers in cardiovascular disease. Ann Transl Med 4: 236, 2016.

21. Bachellerie JP, Cavaillé J and Hüttenhofer A: The expanding snoRNA world. Biochimie 84: 775-790, 2002.

22. Maxwell ES and Fournier MJ: The small nucleolar RNAs. Annu Rev Biochem 64: 897-934, 1995.

23. Tycowski KT, Shu MD and Steitz JA: A mammalian gene with introns instead of exons generating stable RNA products. Nature 379: 464-466, 1996.

24. Pelczar P and Filipowicz W: The host gene for intronic U17 small nucleolar RNAs in mammals has no protein-coding potential and is a member of the 5'-terminal oligopyrimidine gene family. Mol Cell Biol 18: 4509-4518, 1998.

25. Bortolin ML and Kiss T: Human U19 intron-encoded snoRNA is processed from a long primary transcript that possesses little potential for protein coding. RNA 4: 445-454, 1998.

26. Tanaka R, Satoh H, Moriyama M, Satoh K, Morishita Y, Yoshida S, Watanabe T, Nakamura Y and Mori S: Intronic U50 small-nucleolar-RNA (snoRNA) host gene of no protein-coding potential is mapped at the chromosome breakpoint $\mathrm{t}(3 ; 6)(\mathrm{q} 27 ; \mathrm{q} 15)$ of human B-cell lymphoma. Genes Cells 5: 277-287, 2000.

27. Chaudhry MA: Expression pattern of small nucleolar RNA host genes and long non-coding RNA in X-rays-treated lymphoblastoid cells. Int J Mol Sci 14: 9099-9110, 2013.

28. Yu F, Bracken CP, Pillman KA, Lawrence DM, Goodall GJ, Callen DF and Neilsen PM: p53 represses the oncogenic Sno-MiR-28 derived from a SnoRNA. PLoS One 10: e0129190, 2015.

29. Zhao L, Guo H, Zhou B, Feng J, Li Y, Han T, Liu L, Li L, Zhang S, Liu Y, et al: Long non-coding RNA SNHG5 suppresses gastric cancer progression by trapping MTA2 in the cytosol. Oncogene 35: 5770-5780, 2016. 